\title{
Ampliación en la Distribución de Cinco Especies de Murciélagos en Honduras Basada en Detección por Medios Acústicos
}

\author{
Mario Espinal ${ }^{1}$ y José Manuel Mora ${ }^{2}$
}

Resumen. En Honduras, el estudio de los murciélagos ha aumentado, incluido el uso de detectores acústicos. El aporte de los equipos acústicos para la detección de los ultrasonidos de los murciélagos ha sido significativo para incrementar nuestro conocimiento sobre estos mamíferos. Muestreamos la comunidad de murciélagos del municipio de San Marcos de Colón en el sur de Honduras, departamento de Choluteca. Durante cuatro visitas de una semana al sitio trabajamos con el equipo de detección acústica "ANABAT SD2 CF Bat Detector" y logramos la identificación de 24 especies de murciélagos insectívoros. De estos, cinco especies no eran conocidas para el sitio de estudio por lo que su detección representa una ampliación de su ámbito de distribución. Las especies aquí reportadas son Myotis keaysi, Perimyotis subflavus, Lasiurus cinereus (Vespertilionidae), Cynomops mexicanus y Eumops underwoodi (Molossidae).

Palabras clave. Chiroptera, Cynomops mexicanus, ecolocación, detección acústica, Eumops underwoodi, Lasiurus cinereus, Myotis keaysi, Perimyotis subflavus.

\section{Distribution Extension of Five Bat Species in Honduras Based on their Detection by Acoustic Means}

\begin{abstract}
The study of bats has increased in Honduras, including the use of acoustic detectors. The contribution of acoustic equipment for the detection of bat ultrasounds has been significant to increase our knowledge of these mammals. We sampled the bat community in the municipality of San Marcos de Colón in southern Honduras, department of Choluteca. During four a week long visits to the site we used the acoustic detection equipment ANABAT SD2 CF Bat Detector and managed to identify 24 insectivorous bat species. Of these, five species were not known for the study site so its detection represents an extension of its distribution range. The species reported here are Myotis keaysi, Perimyotis subflavus, Lasiurus cinereus (Vespertilionidae), Cynomops mexicanus y Eumops underwoodi (Molossidae).
\end{abstract}

Key words. Acoustic detection, Chiroptera, Cynomops mexicanus, ecolocation, Eumops underwoodi, Lasiurus cinereus, Myotis keaysi, Perimyotis subflavus.

\section{Introducción}

Los murciélagos insectívoros constituyen cerca del $60 \%$ de la fauna quiropterológica neotropical (Simmons 2005). No obstante, a pesar de su importancia numérica, se encuentran poco representados en los inventarios faunísticos (KrakerCastañeda y Pérez 2012, Clarke et al. 2005, MacSwiney et al. 2007). Los muestreos tradicionales con redes de niebla y búsqueda de sitios de percha o descanso no han permitido la documentación de las especies de hábitos insectívoros ni su completo ámbito de distribución. Es por ello que se desconoce la distribución en algunos sitios o regiones de varias especies de murciélagos, particularmente de especies insectívoras. Lo mismo ha sucedido con algunas otras especies incluidos algunos filostomátidos no insectívoros.

Algunas técnicas adicionales de muestreo como otros modos de capturas (trampas), y particularmente los detectores acústicos, han permitido el avance del conocimiento de la distribución y otros aspectos de la biología de varias especies (Mora y López 2012). De esta manera se ha llegado a conocer más de especies que utilizan los estratos superiores de la

\footnotetext{
${ }^{1}$ Investigador Asociado, Centro Zamorano de Biodiversidad, Escuela Agrícola Panamericana, Honduras. Correo electrónico mknorops@gmail.com

${ }^{2}$ Instituto Internacional en Conservación y Manejo de Vida Silvestre (ICOMVIS), Universidad Nacional (UNA), Heredia, Costa Rica. Correo electrónico josemora07@gmail.com
} 
vegetación y que además evaden otros métodos convencionales de muestreo directos, como las redes de niebla y las trampas de arpa, por medio de la ecolocación (Duffy et al. 2000, Berry et al. 2004, PechCanche et al. 2010).

En Honduras se documentaron 226 especies de mamíferos (Marineros y Martínez 1998), de las cuales 105 eran murciélagos. Este número de quirópteros ha sido discutible pero siguiendo a Reid (2009), en el país se documentaron 101 especies hasta el 2009. De 1993 (McCarthy et al. 1993) a 2012 (Mora 2012), no se reportó la existencia de especies adicionales de mamíferos en Honduras. Sin embargo, en los últimos años se han reportado cinco especies adicionales de murciélagos para Honduras y la eliminación de una especie de la lista, para un total de 105 especies (Baird et al. 2012, Mora 2012, Divoll y Buck 2013, Mora y López 2010, Mora et al. 2014). Sin embargo, es poco lo que ha aumentado el conocimiento de la distribución de las especies de mamíferos y particularmente de los murciélagos del país.

En los últimos años ha aumentado el interés por los murciélagos de Honduras desde el punto de vista de conservación y de su conocimiento. El aumento de las instalaciones de producción de energía eólica ha hecho que, por cumplimiento de las leyes y por iniciativa propia de algunas empresas, se estén llevando a cabo inventarios y proyectos de monitoreo de la fauna de murciélagos de Honduras. A la vez, varias personas y grupos interesados en la conservación de los murciélagos han desarrollado acciones que proveen datos e información nueva sobre lo murciélagos de Honduras. Esto ha permitido adquirir un mayor conocimiento sobre la diversidad y la distribución de las especies.

La detección acústica de los sonidos emitidos por los individuos de las especies de murciélagos ha permitido el incremento del conocimiento de estos animales. El registro acústico de especies insectívoras en diferentes hábitats y que tienen una variedad de comportamientos es esencial para crear bibliotecas acústicas (O'Farrell y Miller 1999). En el Neotrópico estas bibliotecas han permitido el reconocimiento de varias especies y así conocer en más detalle la distribución de algunas especies e investigar preferencias en el uso de hábitat (Jung et al. 2007, Ochoa et al. 2000).

\section{Materiales y Métodos}

Muestreamos la comunidad de murciélagos en San Marcos de Colón (Figura 1) con redes de niebla, trampas de arpa y detección acústica durante cuatro visitas al sito en 2014. Se enfatizó la detección de murciélagos insectívoros con el detector ANABAT SD2 CF Bat Detector (Titley Electronics, Australia) a través de un muestreo de forma activa durante las primeras horas de la noche, que son las de mayor actividad de los murciélagos (Mora y López 2012).



Figura 1. Ubicación de los puntos donde se llevaron a cabo las grabaciones de murciélagos insectívoros en el municipio de San Marcos de Colón, departamento de Choluteca, Honduras. Los números indican los sitios de detección de 1. Myotis keaysi, 2. Perimyotis subflavus, 3. Lasiurus cinereus, 4. Cynomops mexicanus, 5. Eumops underwoodi. 
Se hicieron grabaciones que fueron aisladas individualmente para ser comparadas con los espectrogramas conocidos de las especies. Las grabaciones fueron analizadas con el programa AnalookW versión 3.9. La identificación de las especies se basó en la comparación de la estructura (forma) de las vocalizaciones según los trabajos y sugerencias de Barclay (1983), Fenton et al. (1998), Granados (2001), Kalko (1995), O'Farrell y Miller (1997, 1999), Miller (2003), Rydell et al. (2002) y Kraker-Castañeda et al. (2013).

\section{Resultados y Discusión}

El trabajo realizado en San Marcos de Colón ha permitido la detección de 24 especies de murciélagos insectívoros por medio de grabaciones de sus llamados de ecolocación. De estas 24 especies, cinco no eran conocidas del sitio de estudio por lo que su detección significa una aumento del ámbito de distribución conocido.

El mioto de Keay (Myotis keaysi) se distribuye desde el sur de Tamaulipas, México, a través de la península de Yucatán y América Central, hasta el norte de Venezuela, incluida Trinidad y en el oeste de Colombia, sigue las estribaciones de los Andes en el Perú hasta el norte de Argentina (Eisenberg y Redford 1999, Reid 2009). La grabación de Myotis keaysi (Figura 2) en el área de estudio es un registro que representa el tercer reporte documentado y conocido para esta especie en Honduras. Este registro significa una ampliación en su ámbito de distribución en Honduras de $113 \mathrm{~km}$ al sureste desde su localidad conocida, ubicada a $1 \mathrm{~km}$ al oeste de Talanga, departamento de Francisco Morazán (LaVal 1973).

El ámbito de distribución conocido de Perimyotis subflavus se extiende del sureste de Canadá y Estados Unidos hasta Guatemala y el este de Honduras (Simmons 2005, Reid 2009). En Centroamérica se conoce en Guatemala (Carter et al. 1966), Honduras (McCarthy et al. 1993) y Nicaragua (Medina-Fitoria 2014). Registramos a Perimyotis subflavus por medio de una grabación de su llamado (Figura 3) en nuestra área de estudio, lo que amplía su ámbito de distribución en Honduras en aproximadamente $226 \mathrm{~km}$ al suroeste, desde Dulce Nombre de Culmí, Olancho (Rinker 1948), hasta el municipio de San Marcos de Colón en el departamento de Choluteca.

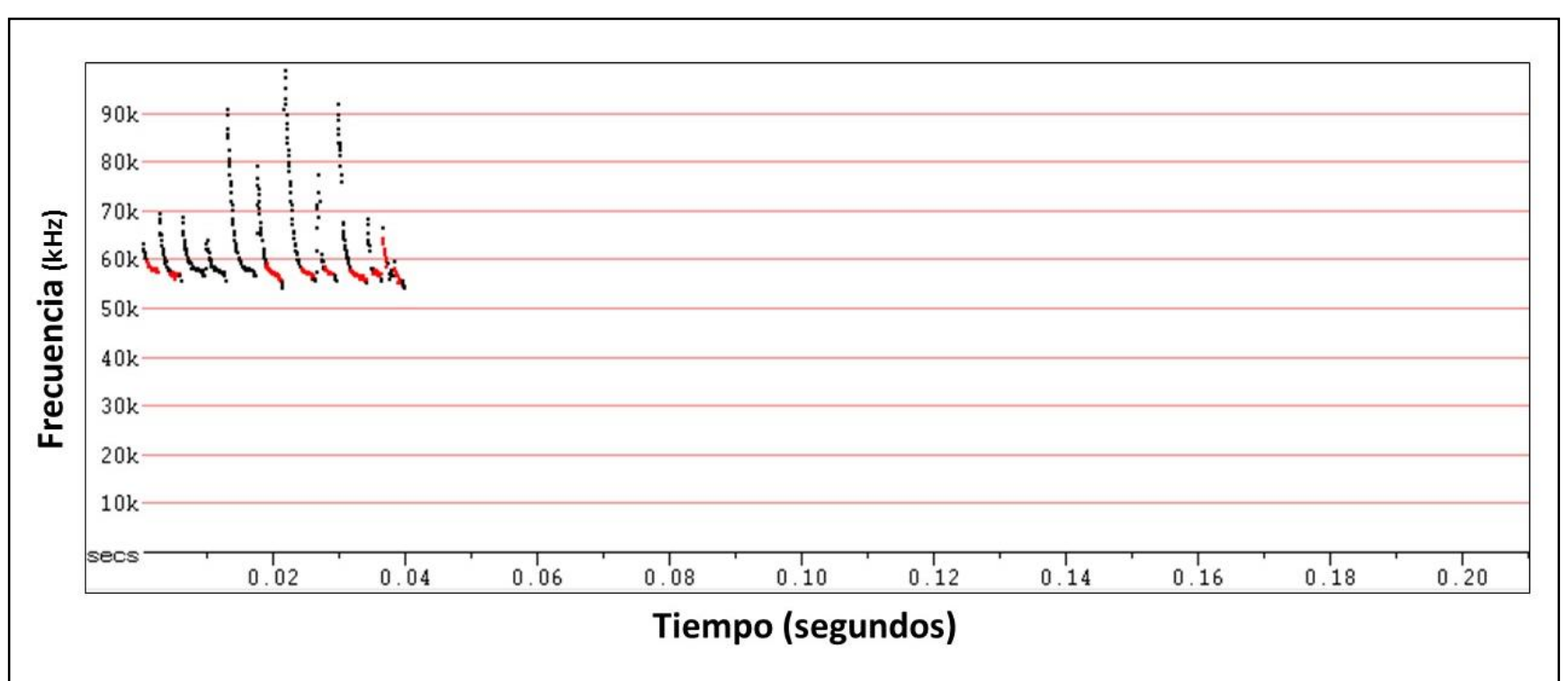

Figura 2. Espectrograma de Myotis keaysi; grabación hecha en San Marcos de Colón en el sur de Honduras. 


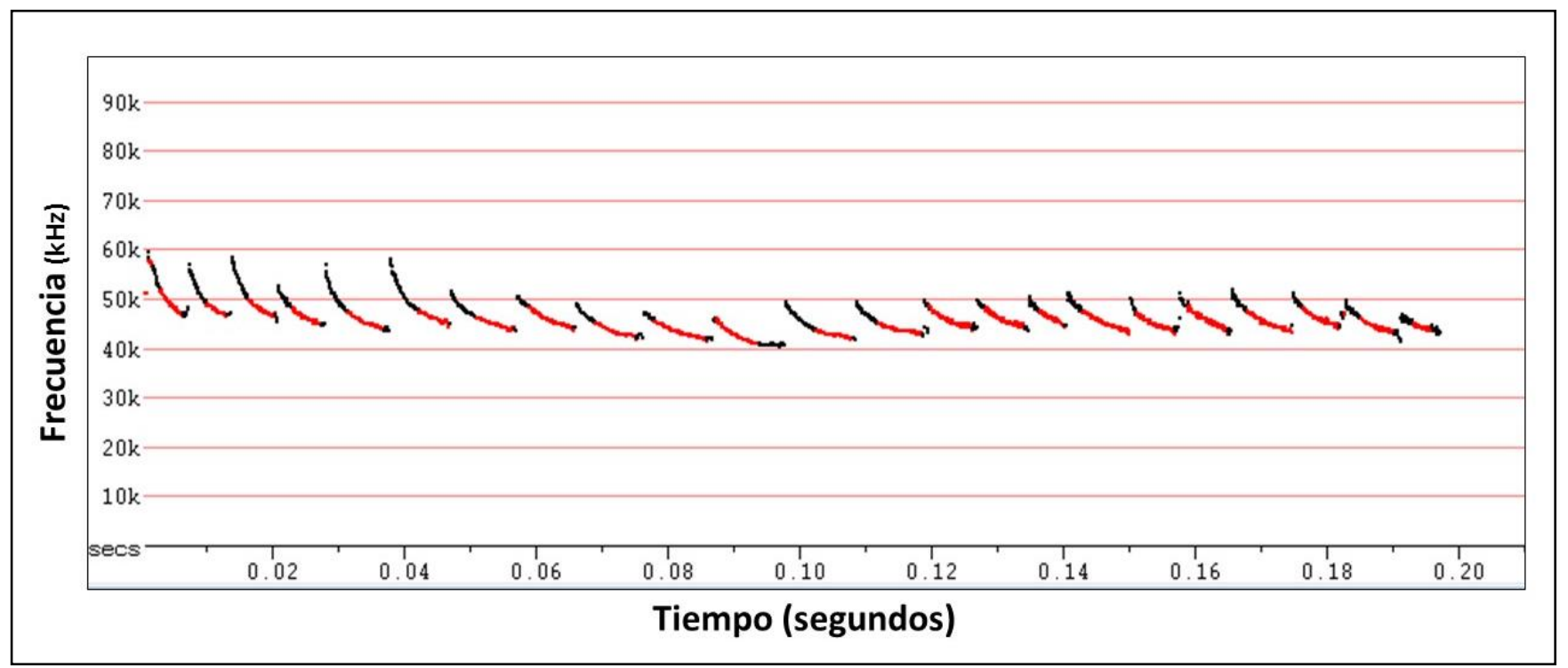

Figura 3. Espectrograma de Perimyotis subflavus; grabación hecha en San Marcos de Colón en el sur de Honduras.

El murciélago canoso (Lasiurus cinereus) tiene una amplia distribución desde Canadá a Chile y varias islas en el Caribe, el Atlántico y el Pacífico, incluso Hawái. La especie parece ser un visitante raro en Centroamérica donde ha sido reportado en Guatemala (Carter et al., 1966), Honduras (Mora y López, 2010), Nicaragua (Medina-Fitoria 2014) y
Panamá (Reid, 2009). Su detección por medios acústicos en San Marcos de Colón (Figura 4) representa una ampliación de su ámbito de distribución en Honduras de aproximadamente 87 km al sureste. Este reporte es importante pues es apenas el segundo de Lasiurus cinereus en Honduras.

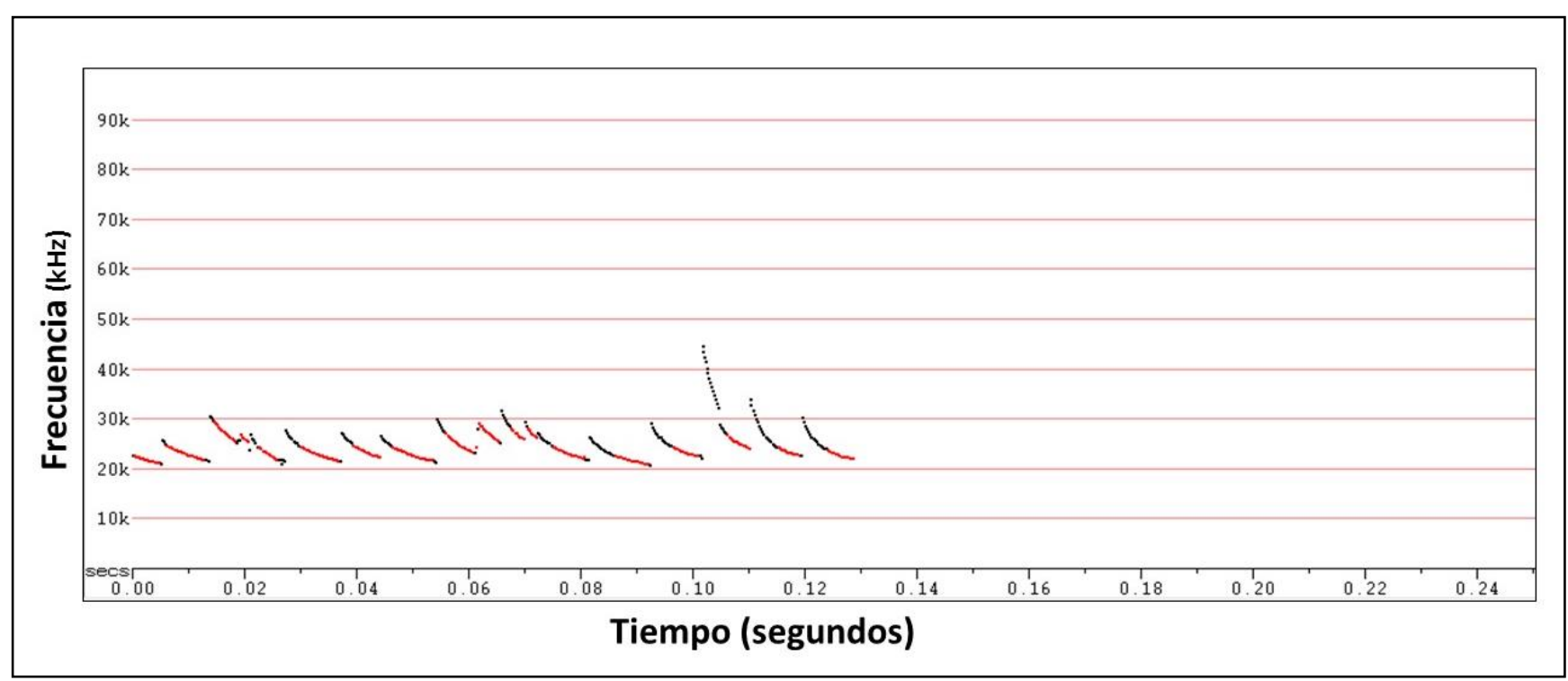

Figura 4. Espectrograma de Lasiurus cinereus; grabación hecha en San Marcos de Colón en el sur de Honduras. 
El murciélago cara de perro mexicano (Cynomops mexicanus) se distribuye desde la costa oeste de Nayarit, México hasta Ecuador, Venezuela, Guyanas y NE de Brazil, extendiéndose hasta Trinidad (Simmons 2005). C. mexicanus, previamente incluido en la lista de mamíferos de Honduras como Molossops greenhalli (McCarthy et al. 1993), fue reclasificado posteriormente por Peters et al. (2002). La grabación de su llamado (Figura 5) para este reporte constituye el tercer registro para esta especie en el país y extiende su ámbito de distribución 143 km al sureste del departamento de Comayagua hasta la comunidad de Jícaro Largo en el municipio de San Marcos de Colón, en el departamento de Choluteca.

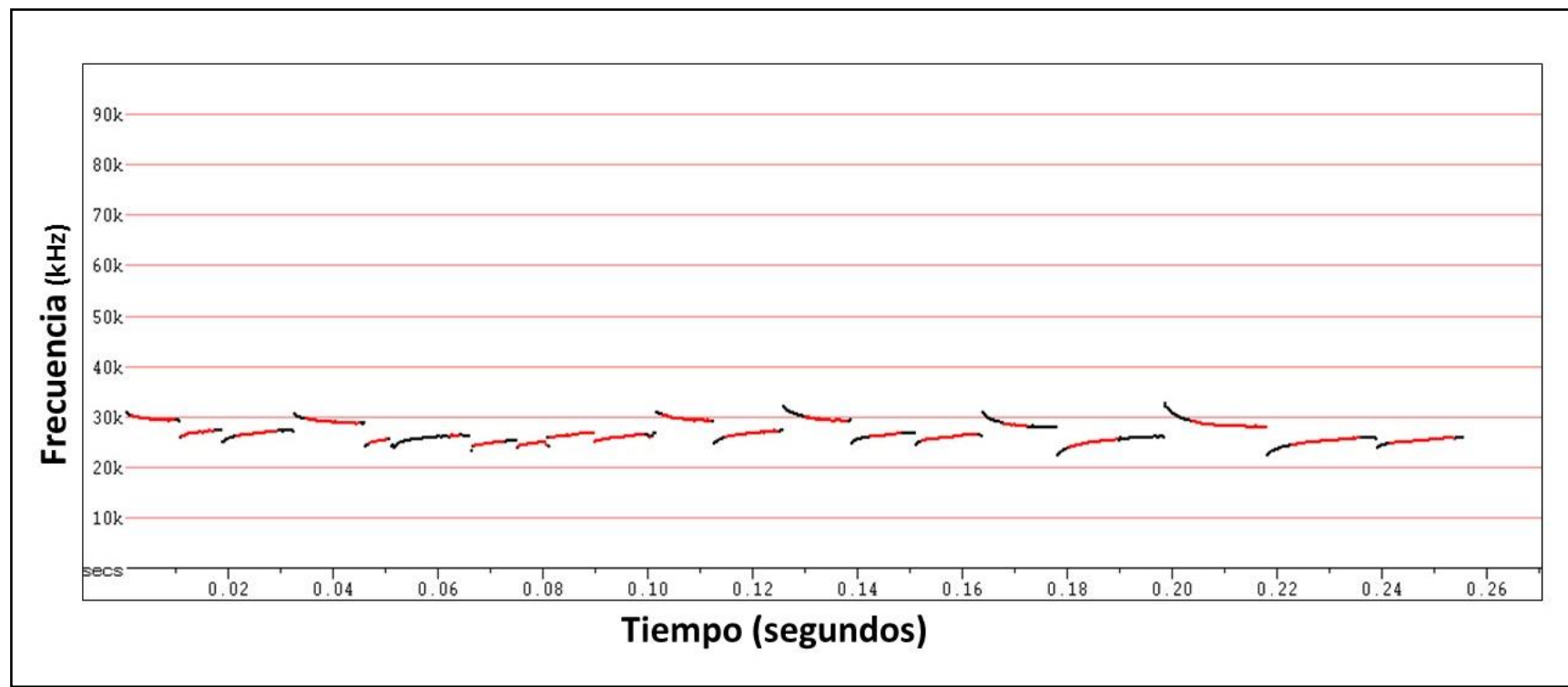

Figura 5. Espectrograma de Cynomops mexicanus; grabación hecha en San Marcos de Colón en el sur de Honduras.

El eumopo de Underwood (Eumops underwoodi) se distribuye desde Arizona hasta Nicaragua (Hall 1981, Simmons 2005). La detección acústica en el sur de Honduras de E. underwoodi (Figura 6) constituye apenas el segundo registro de esta especie para el país. A la vez este registro significa una extensión del ámbito de distribución de E. underwoodi de $148 \mathrm{~km}$ al sureste de la otra localidad conocida, $6 \mathrm{~km}$ al norte de Chinacla en el departamento de la Paz (McCarthy et al. 1993).

Los parámetros cualitativos y cuantitativos de los llamados de ecolocación de Myotis keaysi, Cynomops mexicanus y Eumops underwoodi registrados en la presente trabajo, son similares a los reportados para estas especies por Miller (2003) en el noroeste de Belice y por Kraker-Castañeda (2010) en Guatemala. Los llamados de Lasiurus cinereus y Perimyotis subflavus concuerdan con los registrados para estas especies por Rougeaux (2013) y O'Farrell et al. (2002) en los Estados Unidos. Cabe destacar que Thomas et al. (1987) identificaron al murciélago canoso (Lasiurus cinereus) como la especie que muestra la variación más extrema de los parámetros medidos de llamadas de ecolocación.

Otras especies que hemos registrado mediante detección acústica en el sitio de trabajo son: Peropteryx kaplerii, Mormoops megalophylla, Pteronotus parnellii, Pteronotus davyi, Pteronotus gymnonotus, Eptesicus furinalis, Eptesicus fuscus, Lasiurus blossevillii, Lasiurus ega, Lasiurus intermedius, Myotis nigricans, Rhogesssa tumida, Molossus molossus, Molossus rufus, Molossus sinaloae, Nyctinomops laticaudatus y Tadarida brasiliensis. Además de otras dos especies no identificadas aún. 


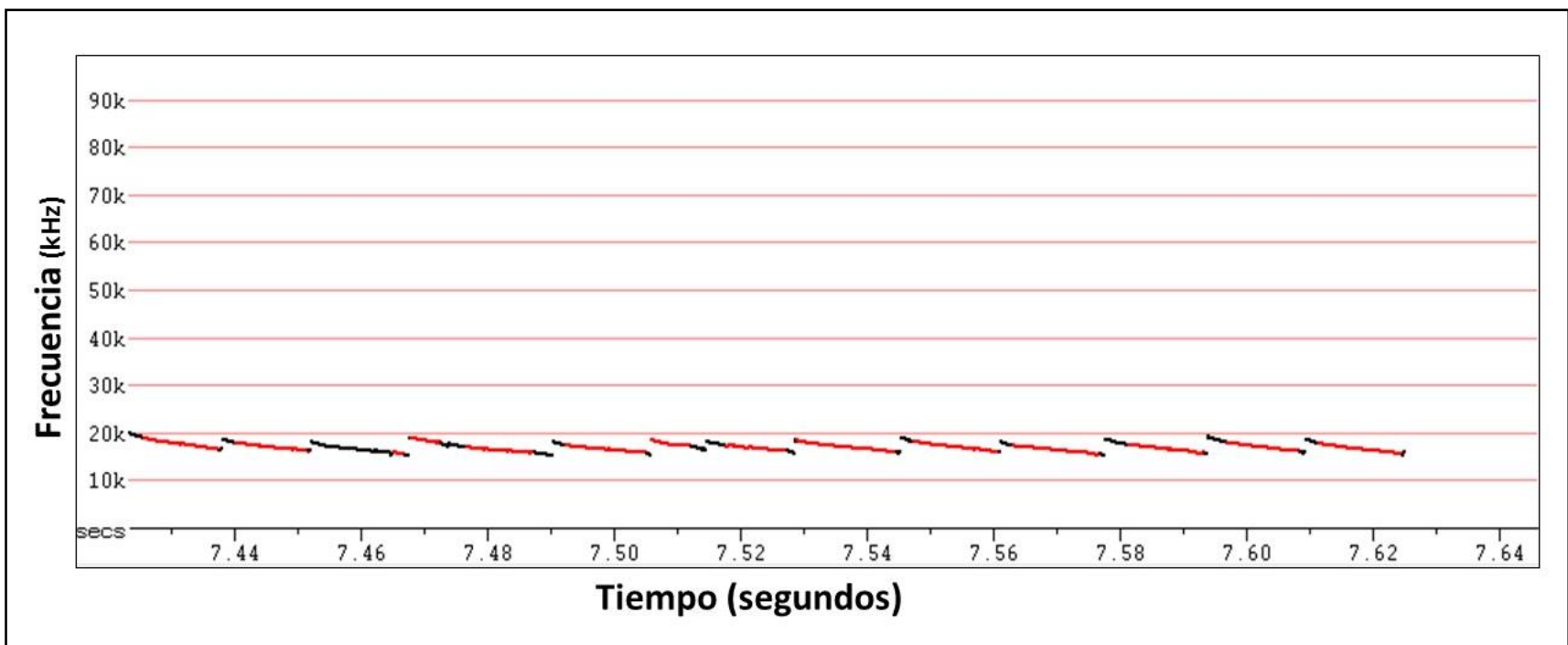

Figura 6. Espectrograma de Eumops underwoodi; grabación hecha en San Marcos de Colón en el sur de Honduras.

El registro exclusivamente acústico de Myotis keaysi, Perimyotis subflavus, Lasiurus cinereus, Cynomops mexicanus y Eumops underwoodi en la presente nota, evidencia la importancia de realizar estudios que utilicen una variedad de métodos de detección combinados y que incluyan la detección ultrasónica. En la medida que el número de este tipo de estudios se incremente, tendremos más información para completar los inventarios, entender las preferencias de hábitat y la distribución de especies de murciélagos hasta ahora poco conocidas y aumentar nuestro conocimiento sobre la distribución real de las especies.

Agradecimientos. Agradecemos la colaboración prestada por todas aquellas personas que han participado en este trabajo en los muestreos de campo o facilitando información. Agradecimiento en especial a Carlos O'reilly, Rodie Ordoñez, Hermes Vega, Arnulfo Medina Fitoria, Octavio Saldaña y Carlos Funes.

\section{Literatura Citada}

Baird A.B, M.R. Marchán-Rivadeneira, S.G. Pérez y R.J. Baker. 2012. Morphological Analysis and Description of Two New Species of Rhogeessa (Chiroptera: Vespertilionidae) from the Neotropics. Occasional Papers of the Museum of Texas Tech University, Number 307. $32 \mathrm{p}$.

Barclay, R.M.R. 1983. Echolocation calls of emballonurid bats from Panama. Journal of Comparative Physiology 151(4):515-520.

Berry, N., W. O'Connor, M.W. Holderied y G. Jones. 2004. Detection and avoidance of harp traps by echolocating bats. Acta Chiropterologica 6(2):335-346.

Carter, D.C., R.H. Pine y W.B. Davis. 1966. Notes on Middle American bats. Southwestern Naturalist 11(4):488-499.

Clarke, F.M., D.V. Pio y P.A. Racey. 2005. A comparison of logging systems and bat diversity in the Neotropics. Conservation Biology 19(4):1194-1204.

Divoll, T.J. y D.G. Buck. 2013. Noteworthy field observations of cave roosting bats in Honduras. Mastozoología Neotropical 20(1):149-151. 
Duffy, A.M., L.F. Lumsden, C.R. Caddle, R.R. Chick y G.R. Newell. 2000. The efficacy of Anabat ultrasonic detectors and harp traps for surveying microchiropterans in southeastern Australia. Acta Chiropterologica 2(2):127-144.

Eisenberg, J.F. y H. Redford. 1999. Mammals of the Neotropics, the central Neotropics. Volumen 3. University of Chicago Press, Illinois, EUA. 624 p.

Fenton, M.B., I.L Rautenbach, J. Rydell, H.T. Arita, J. Ortega, S. Bouchard, L.B. Hovorka, E. Odgren, C.V. Portfors, W.M. Scully, D.M. Syme y M.J. Vonhof. 1998. Emergence, echolocation, diet and foraging behavior of Molossus ater (Chiroptera: Molossidae). Biotropica 30(2):314-320.

Granados, H.J. 2001. Los sonidos de ecolocalización de los murciélagos insectívoros de Yucatán. Tesis de Licenciatura. Instituto de Ecología, Universidad Autónoma de México. $72 \mathrm{p}$

Hall, E.R. y K.R. Kelson. 1981. The mammals of North America. Volume 1. Segunda edición. John Wiley and Sons, New York, EUA. 546 p.

Jung, K., E.K.V. Kalko y O. von Helversen. 2007. Echolocation calls in Central American emballonurid bats: signal design and call frequency alternation. Journal of Zoology 272(2):125-137.

Kalko, E.K.V. 1995. Echolocation signal design, foraging habitats and guild structure in six Neotropical sheathtailed bats (Emballonuridae). In P.A. Racey y S.M. Swift (Eds.). Ecology, Evolution and Behavior of Bats. The Zoological Society of London, Oxford. Inglaterra. p 259273.

Kraker-Castañeda, C. 2010. Análisis de la alimentación de murciélagos insectívoros en cultivos de café del departamento de Sacatepéquez. Informe Final. FODECYT No.12-2008. Guatemala. 93 p.

Kraker-Castañeda y S. Pérez. 2012. Detección ultrasónica de murciélagos insectívoros en cafetales de La Antigua Guatemala, Guatemala. Revista Científica Instituto de Investigaciones Químicas y Biológicas 22(1):43-53.

Kraker-Castañeda, C. A. Santos-Moreno y J.L. GarcíaGarcía. 2013. Riqueza de especies y actividad relativa de murciélagos insectívoros aéreos en una selva tropical y pastizales en Oaxaca, México. Mastozoología Neotropical 20(2):255-267

LaVal, R.K. 1973. A revision of the Neotropical bats of the genus Myotis. Bulletin of the Natural History Museum of Los Angeles County 15:1-54.

MacSwiney, M.C., P. Vilchis, F.M. Clarke y P.A. Racey. 2007. The importance of cenotes in conserving bats assemblages in the Yucatan, Mexico. Biological Conservation 136(4):499-509.

Marineros, L. y F. Martínez. 1998. Mamíferos de Honduras. INADES. Tegucigalpa. 374 p.
McCarthy, T.J., W.B. Davis, J.E. Hill, J.K. Hill, J.K. Jones Jr. y G.A. Cruz. 1993. Bat (Mammalia: Chiroptera) records, early collectors, and faunal lists for northern Central America. Annals of Carnegie Museum 62(3):191-228.

Medina-Fitoria, A. 2014. Murciélagos de Nicaragua. Guía de campo. Programa para la conservación de los murciélagos de Nicaragua (PCMN) y Ministerio del Ambiente y los Recursos Naturales (MARENA). Managua, Nicaragua. 278 p.

Miller, B.W. 2003. Community ecology of the nonphyllostomid bats of Northwestern Belize, with a landscape level assessment of the bats of Belize. Tesis de Doctorado. University of Kent at Canterbury, Reino Unido. $294 \mathrm{p}$

Mora, J.M. 2012. Big Red Bat Lasiurus egregius (Vespertilionidae) in Honduras. The Southwestern Naturalist 57(1):104-105.

Mora, J.M., L. Marineros y L.I. López. 2014. First record of the striped yellow-eared bat, Vampyriscus nymphaea, (Stenodermatinae, Phyllostomidae) in Honduras. Caribbean Journal of Science 48(1):49-51

Mora, J.M y L.I. López. 2010. First Record of The Hoary Bat (Lasiurus cinereus, Vespertilionidae) for Honduras. Ceiba 51(2):89-90.

Mora, J.M. y L.I. López. 2012. Protocolo monitoreo de murciélagos. Instituto Nacional de Conservación y Desarrollo Forestal, Áreas Protegidas y Vida Silvestre. Honduras. $50 \mathrm{p}$.

Ochoa, J., M.J. O'Farrell y B.W. Miller. 2000. Contribution of acoustic methods to the study of insectivorous bat diversity in protected areas from northern Venezuela. Acta Chiropterologica 2(2):171-183.

O'Farrell, M.J. y B.W. Miller. 1997. A new examination of echolocation calls of some Neotropical bats (Emballonuridae y Mormoopidae). Journal of Mammalogy 87(3):954-963.

O'Farrell, M.J., C. Corben y W.L. Gannon. 2000. Geographic variation in the echolocation calls of the hoary bat (Lasiurus cinereus). Acta Chiropterologica 2(2):185-196.

O'Farrell, M.J. y B.W. Miller. 1999. Use of vocal signatures for the inventory of free-flying Neotropical bats. Biotropica 31(3):507-516.

Pech-Canche, J. MacSwiney, M.C. y E. Estrella. 2010. Importancia de los detectores ultrasónicos para mejorar los inventarios de murciélagos Neotropicales. Therya 1(3): 227-234.

Peters, S.L., L.K. Lim y M.D. Engstrom. 2002. Systematics of dog-faced bats (Cynomops) based on molecular and morphometric data. Journal of Mammalogy 83(4):10971101.

Reid, F. A. 2009. A field guide to the mammals of Central America and Southeast Mexico. Segunda edición. Oxford University Press. New York, EUA. 346 p. 
Rinker, G.C. 1948. A bat (Pipistrellus) recorded from Honduras. Journal of Mammalogy 29(2):179-180.

Rougeaux, J. 2013. Species specific bat activity \& susceptibility to White-nose Syndrome. McCrady SCARNG Training Center Eastover. South Carolina, EUA. 66 p.

Rydell, J., H.T. Arita, M. Santos y J. Granados. 2002. Acoustic identification of insectivorous bats (Order Chiroptera) of Yucatan, Mexico. Journal of Zoology 257(1):27-36.
Simmons, N.B. 2005. Order Chiroptera. In D.E.Wilson y D.M. Reeder (Eds.). Mammal species of the world: a taxonomic and geographic reference. Tercera edición. Smithsonian Institution Press, Washington, D.C. EUA. p. 312-529.

Thomas, D.W., G.P. Bell y M.B. Fenton. 1987. Variation in echolocation call frequencies recorded from North American vespertilionid bats: a cautionary note. Journal of Mammalogy 68(4): 842-847.

Recibido para publicación el 2 de abril de 2015.

Aceptado para publicación el 18 de mayo de 2015. 